\title{
Numerical simulation of heat transfer and temperature distribution in a printed circuit board enclosure model for different geometries and Reynolds numbers
}

\author{
S. Varela ${ }^{1}$, G. Usera ${ }^{2}$, A. Vernet ${ }^{1} \&$ J. A. Ferré \\ ${ }^{1}$ Department of Mechanical Engineering, \\ Universitat Rovira i Virgili, Spain \\ ${ }^{2}$ Instituto de Mecánica de los Fluidos, Facultad de Ingeniería, \\ Universidad de la República, Uruguay
}

\begin{abstract}
The heat transfer analysis and temperature distribution inside printed circuit board (PCB) enclosures for different geometries was investigated in this paper. Herein, the computational fluid dynamic (CFD) solver caffa3d.MB was employed to simulate the 3D incompressible Navier-Stokes equations. The numerical method uses a spatial discretization based on block-structured, nonorthogonal body fitted grids. Numerical simulations for two different PCB geometry configurations are analysed using eight Reynolds numbers. The temperature field is practically the same above the plate encasing the PCB, differences appear below the plate. While analysing the plane just below for the open geometry the main consideration is the presence of a hot zone. The greater heat transfer is obtained in the top channel for both geometries. In turn, in the bottom channel the heat transfer is greater in the closed geometry than the open one. The calculating results show that the cooling of the PCB is more efficient for the closed geometry.

Keywords: electrical enclosure, caffa3d.MB, numerical simulation, heat transfer.
\end{abstract}




\section{Introduction}

A large number of experimental and numerical fluid flow analyses in complex domains can be found in the literature. These systems become more complicated when the heat transport effects are included [2-4]. This is the case of the refrigeration of the printed circuit boards (PCB). The PCBs are electronic circuits that generate heat by Joule effect and need to be refrigerated, usually by air. This is a problem of fluid flow in complex domain where we can find the coexistence of laminar and turbulent fluid regions and heat transfer effects by natural/forced convection, conduction and radiation. Difficulty grows by the fact that there is a trend to reduce the size of the PCB enclosures to fit the needs of the manufactures. This increases the warming problems and is the cause of a reduction of the PCB's efficiency and lifespan. PCBs can be found in a large number of the daily used devices as computers, automobiles [5] or airplanes, and also in less common applications as diverse elements of spacecraft and telecommunication satellites.

The main objective is to analyze the heat transfer and temperature distribution in PCB enclosed cavities with different geometries

A deeper understanding of flow structures and temperature field distribution can lead to a better enclosure design improving heat dissipation of the PCBs.

\section{Physical model}

The system consists of a rectangular box with two square section openings of side $h=0.024 \mathrm{~m}$ working as inlet and outlet for the circulating fluid.

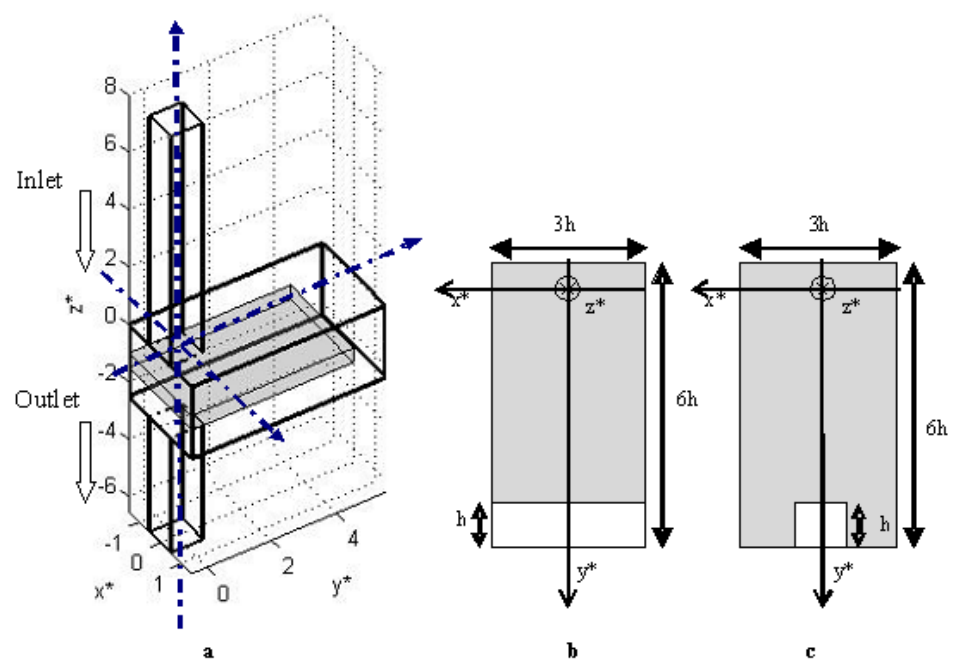

Figure 1: Model's sketch, a three dimensional view, b and c Top view of the two studied models. 
The flow entrance's cavity is carried out through a square base block of $h \times h$ and $8 h$ long, outlet block has the same base dimensions and $4 h$ long. The inlet block is longer than the outlet one to minimize perturbations. A schematic representation of the PCB system is shown in fig. 1a.

The size of the housing is $6 h \times 3 h \times 2.5 h$ with a central plate simulating the PCB placed in the box's symmetry plane. Two different geometries of this central plate were used in this study.

The first one is a rectangular prism $3 h$ wide, $0.5 h$ high and $5 h$ long that was studied before by Usera et al. [6] The second case corresponds to a rectangular prism $3 h$ wide, $0.5 h$ high and $6 h$ long with an $h \times h$ opening. The junction box model can be considered as the sum of two channels, the top and the bottom ones with size of $6 h$ long, $3 h$ wide and $h$ tall. To help differentiate among configurations we refer to the first one (fig. 1b) as the open geometry and the second (fig. 1c) as the closed geometry.

\section{Numerical method}

Caffa 3 d.MB is an original Fortran implementation [8] of a fully finite volume method for solving the 3D incompressible Navier-Stokes equations in complex geometry. This three-dimensional solver is based on a two-dimensional solver described by Ferziger and Pèric [1]. Spatial discretization is based on blockstructured, non-orthogonal, body fitted; collocated grids with first order (UDS) and second order (CDS) schemes for the convective term. Improved linear interpolation schemes for non-orthogonal grids are also included following Lehnhauser and Schafer's work [9]. For the time discretization, fully implicit two-level first order (implicit backward Euler) and three-level second order schemes are available. The SIMPLE algorithm is implemented for the coupling between velocity and pressure [1]. Different interfaces between grids blocks are currently supported, among which only one-to-one interfaces are use here. Domain decomposition on the basis of grid blocks is applied through OpenMP, with current simulation running on dual processor machines. A full description of the flow solver can be found in [8]. Validation of the flow solver against experimental data and benchmark simulations for different cases can be found also in $[8,10,11]$ and for the present system in greater detail in [6]. The mathematical model comprises the mass and momentum balance equations for an incompressible Newtonian fluid with constant properties and the Boussinesq approximation for buoyancy effects due to temperature induced small density variations. In our case the heat equation was introduced in the model by the conservation law for a generic passive scalar. In order to determine the degree of mesh refinement needed to achieve a proper fit to resolve the momentum and temperature boundary layer, simulations were performed for different degrees of refinement. A refined grid with a spatial resolution set to $h / 65$ near the walls and about $h / 40$ at the core had been used in the simulations for all cases studied [14].

Reynolds number defined by $R e=U_{0} h \rho / \mu$ was set ranging from $\operatorname{Re}=100$ to $\mathrm{Re}=10000$. Table 1 shows the select $\mathrm{Re}$ number, corresponding to mean 
velocity at the inlet region, respectively. The flow is laminar up to around $\mathrm{Re}=5000$, from that value the flow behaviour becomes turbulent [6].

Table 1: Different Reynolds numbers corresponding to mean velocity at the inlet region.

\begin{tabular}{|c|c|c|c|c|c|c|c|}
\hline $\mathbf{R e}$ & 300 & 600 & 1160 & 3000 & 5790 & 8000 & 10000 \\
\hline $\mathbf{U}_{\mathbf{0}}(\mathbf{m} / \mathbf{s})$ & 0.012 & 0.024 & 0.048 & 0.12 & 0.24 & 0.32 & 0.41 \\
\hline
\end{tabular}

The LES turbulence model has been used in the simulations for the highest Re. The chosen values of the relaxation coefficients joined with a cycle of 10 outer iterations allowed to ensure a reduction of the convergence parameter in 3 orders of magnitude at each time step. These convergence levels are adequate to analyse the state of flow strongly governed by the boundary conditions. All numerical model details can be found in [14]. In all cases the hot temperature was $T_{h}=30^{\circ} \mathrm{C}$ (plate temperature) while cold and reference temperature (fluid temperature) were $T_{c}=T_{r e f}=20^{\circ} \mathrm{C}$. This $\Delta T$ was selected as a first step to investigate their influence on the heat transfer and flow structures. Moreover, the application of the Boussinesq approximation has minor effects on the solution. For greater values of $\Delta T$ the calculations have to be obtained employing fluid properties as function of temperature [12] thus constant physical fluid properties were considered. The dimensionless time step was $d t /\left(h / U_{0}\right)=5 \times 10^{-3}$ and the simulations have been ran starting from null velocity fields through $11 \times 10^{3}$ time steps, or about $55 \mathrm{~s}$ of flow time. Throughout the simulations the flow exhibits non-stationary characteristics but reaching a regime state independent of initial conditions. The mean fields presented below were averaged over a time period that did not include the start time. Numerical simulation yields instantaneous 3D information of dynamic and temperature fields in the PCB model. Thus, the three velocity components and temperature profiles can be obtained at top, bottom and passage channels of the model simultaneously.

\section{Results and discussion}

The dynamic field shows that the flow structures present in the top channel are a pair of quasi-symmetric counter rotating vortices for both geometries. In the bottom channel the flow behaviour depends on the geometry, for the closed one it remains the pair of quasi-symmetric counter rotating vortices while in the open geometry these structures disappear giving way to smaller ones [14].

\subsection{Temperature fields}

In the top channel the fluid is scarcely influenced by the plate temperature except at very close distances from it. Both in the passage and bottom channel the fluid is more influenced by the temperature of the plate, but this influence only represents a fluid temperature rise in $1^{\circ} \mathrm{C}$ in areas not close from the plate. This behaviour is similar for different $R e$, having more fluid whose temperature raises 
at least $1{ }^{\circ} \mathrm{C}$ for lower Re numbers and less for higher ones but these variations are not really significant at a global level. The greatest influence of the plate temperature in the fluid is presented very close from it so mean temperature fields in the plane $\left(x^{*}, y^{*}\right)$ at the cell points in the $z^{*}$ direction nearest from the plate are studied. These planes are located at $h / 65$ of the plate, both in the top and the bottom channel. For the all considered Reynolds numbers, the lower temperature zone coincides with the entrance area of the fluid in the cavity having a difference in temperature values for each $R e$. For $R e=300$, the fluid temperature is practically the same as the plate along the entire cavity having a little variation in the proximity of the passage channel. For $R e=1160$ and $R e=5790$ the areas with a higher temperature change match the location of the larger counter-rotating vortices. For the open geometry, with the same setting described above, the mean temperature field is the same as the one obtained for the closed geometry at least until the passage channel.

\subsection{Bottom channel}

The biggest differences in the mean temperature distribution appear in the bottom side of the plate for both geometries. The small, weaker, vortical structures present in the open geometry result in a higher values of temperature field along the channel (fig. 2).

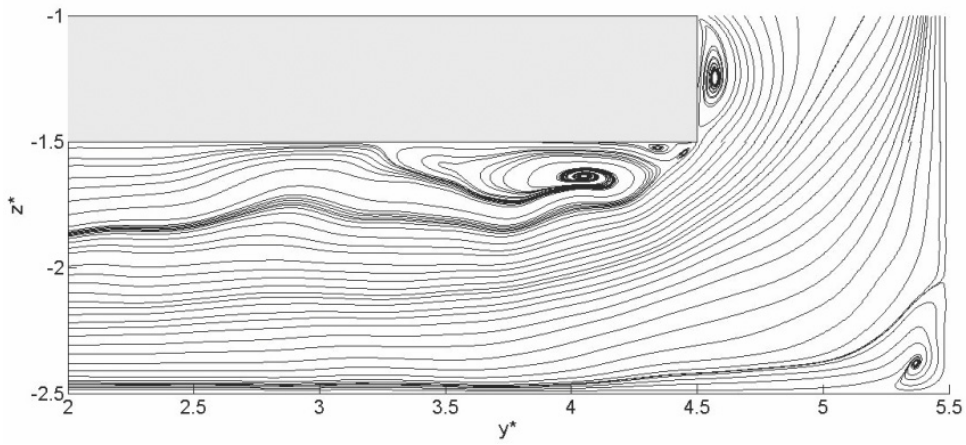

Figure 2: Open geometry, $\mathrm{Re}=5790$. Mean velocity field streamlines throughout the cavity. Plane $\left(\mathrm{z}^{*}, \mathrm{y}^{*}\right)$ at $\mathrm{x}^{*}=0$.

For this geometrical configuration for $R e=5790$ a hot region located near the passage channel at $y^{*}=4.2$ is detected. Figure 2 shows the streamlines for the mean velocity field for $R e=5790$ which is characterized, in the bottom channel just below the plate, by a strong recirculation downstream from the leading edge of the plate [6] matching with the hot zone observed in fig. 3a. If the instantaneous flow was to be permanently as picture in fig. 2 the heat transfer from the plate near the recirculation region could be adversely affected in addition to generating undesired localized mechanical wear. That recirculation is present only in the open geometry and for all Reynolds numbers greater than 
1160. Moreover, a higher temperature spot near the output region $\left(y^{*}=0.6\right)$ is observed. These hot spots increase the PCB's warming problems and therefore could reduce their efficiency and lifespan. For the closed geometry two large vortices, are generated by the jet impinging onto the bottom channel, that has a lower temperature $\left(T_{c}=20^{\circ} \mathrm{C}\right)$.
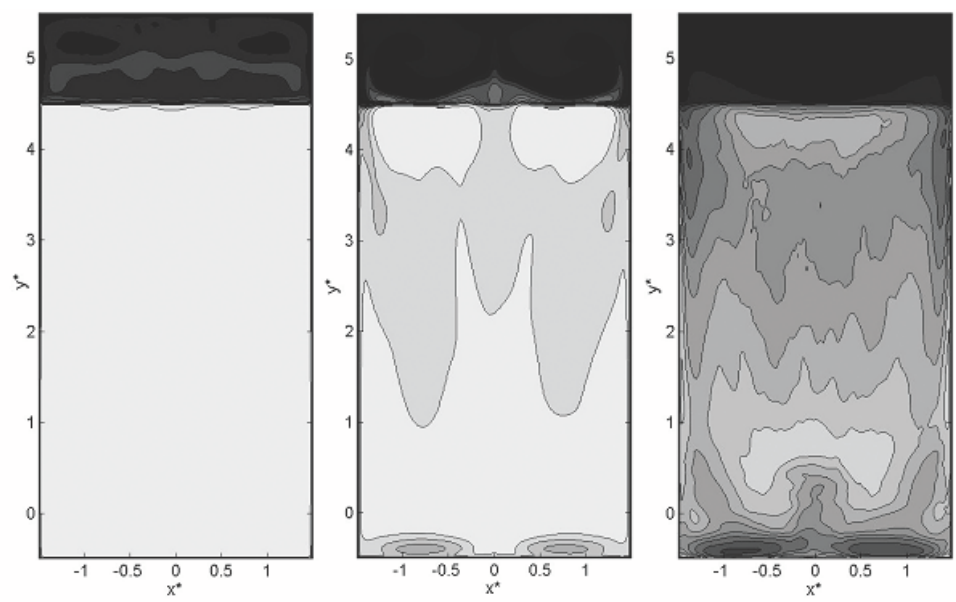

$\mathbf{a}$
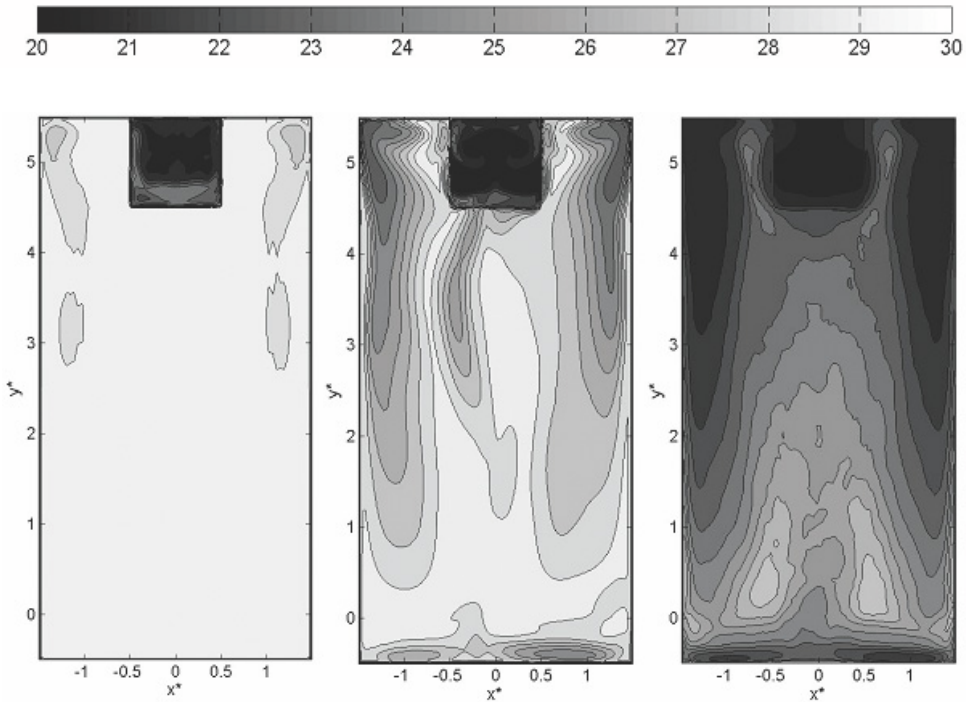

b

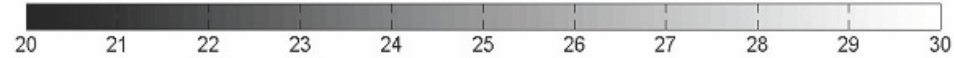

Figure 3: Mean temperature filed, bottom channel. Plane $\left(x^{*}, y^{*}\right)$ for $\mathrm{Re}=300$ (left), $\mathrm{Re}=1160$ (middle) and $\mathrm{Re}=5790$ (right) with temperature difference between the plate and the fluid of $\Delta \mathrm{T}=$ $10^{\circ} \mathrm{C}$. a Open geometry; b Closed geometry. 
These flow structures in the bottom channel are responsible for decreasing the temperature in some regions of the lower side of the plate (fig. $3 \mathrm{~b}$ ). For $\mathrm{Re}=$ 1160 and $\operatorname{Re}=5790$ the lower temperature coincides with the vortices location (about $\mathrm{x}^{*}= \pm 1.25$ ) while the higher temperatures are located in the channel output zone $\left(\mathrm{y}^{*}=0.5\right)$. As found for the open geometry, for $\mathrm{Re}=5790$ higher temperature spot near the output region $\left(\mathrm{y}^{*}=0.6\right)$ is observed although localized. From the temperature contours plotted in fig. 3 , it can be stated that the cooling of the PCB is more efficient for the closed geometry. For both geometries the top channel shows a similar behaviour, but for the bottom channel the open geometry depicts hot spots that could generate problems on the PCB's operation.

\section{Heat transfer}

A good way to study the temperature influence in the system is through the Nusselt number. The Nusselt number is one of the most important parameter in convective heat transfer problems. Convection intensity is determined by the value of Nusselt number. The average Nusselt number of plate is calculated in order to realize the heat transfer phenomenon around the heat source. As we know the heat transfer by convection is, $q=H A \Delta T$, where $H$ is convective coefficient, $A$ is the contact area and $\Delta T$ is the difference of temperature. The heat exchange between fluid and solid boundary:

$$
\frac{q}{A}=H\left(T_{p}-T_{\infty}\right)=-k_{f}\left(\frac{\partial T}{\partial z}\right)_{z=1,-1.5}
$$

where $k_{f}$ is the conduction coefficient of fluid, $T_{\infty}$ is the inlet temperature, $T_{p}$ is the plate temperature, the partial derivation is evaluated in the plate's upper wall $(z=-1)$ and in the plate's bottom wall $(z=-1.5)$. The definition of Nusselt number:

$$
N u=\frac{H h}{k_{f}}=\frac{h\left[\frac{\partial\left(T_{p}-T\right)}{\partial z}\right]_{z=-1,-1.5}}{T_{p}-T_{\infty}}
$$

\subsection{Nusselt vs. Reynolds}

The mean Nusselt number $(\mathrm{Nu})$ variation as a function of Reynolds number for both geometries has been studied. The mean Nusselt number values are obtained, as the temperature fields, in the plane $\left(x^{*}, y^{*}\right)$ parallel to the plate at $h / 65$ of this, both in the top and the bottom channel. In all cases numerical results are presented, considering constant physical properties and the temperature difference between the plate and the fluid of $\Delta T=10^{\circ} \mathrm{C}$. The mean Nusselt number is calculated by averaging in two directions $\left(x^{*}\right.$ and $\left.y^{*}\right)$. In the closed geometry the area used to obtain such mean value is $13 \%$ higher than in the open geometry.

Figure 4 shows the behaviour of $N u$ as function of $R e$ in the closed and the open geometry, both in the top and the bottom channel. 
Where it can be seen that the heat transfer $(\mathrm{Nu})$ is greater in the top channel than that in the bottom one for both geometries, for all Re studied. It can be observed, for the open geometry, that the difference between the two channels is much higher as shown in fig. 4. By comparing the behaviour of $N u$ as function of $R e$ in the top channel for both geometries we can observe that for low $R e$ the heat transfer yield is $5 \%$ higher in the closed geometry than in the open one, for $R e=$ 1160 the heat transfer remains the same for both geometries. While for higher $\mathrm{Re}$, the heat transfer is $2 \%$ higher in the open geometry than in the closed one. In the bottom channel, for all values of $R e$ the heat transfer is larger for the closed geometry than for the open one.

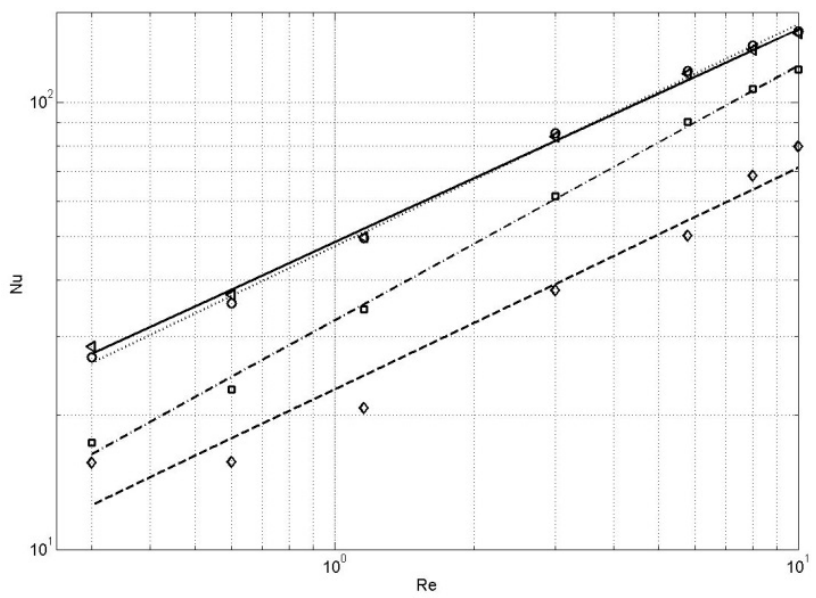

Figure 4: Mean Nusselt number vs. Re at $\Delta \mathrm{T}=10^{\circ} \mathrm{C}$ and its correlations for the closed geometry in top $(\varangle,-)$ ) and bottom $(\square,-\cdot)$ channel.For the open geometry in top $(\circ, \ldots \ldots)$ and bottom $(\diamond,----)$.

Being these differences $10 \%$ higher for low values of Re, up to $80 \%$ for higher Re ones, as can be seen in fig. 4. A general correlation form to obtain the Nusselt number as function of Reynolds and Prandtl numbers is:

$$
N u=C \operatorname{Re}^{\gamma} \operatorname{Pr}^{\beta}
$$

Table 2: Correlation Re and Pr exponents.

\begin{tabular}{|c|c|c|c|}
\hline Geometry & Channel & $\gamma$ & $\beta$ \\
\hline Open & top & 0.5 & 1.98 \\
\hline Open & bottom & 0.49 & 1.60 \\
\hline Closed & top & 0.48 & 1.99 \\
\hline Closed & bottom & 0.57 & 1.79 \\
\hline
\end{tabular}


The value of the constant is $C=1$. Noting the correlation coefficients verifies that in the top channel scarcely any differences observed between both geometries.

\subsection{Nusselt vs. $\mathrm{y}^{*}$}

In this section the behaviour of mean Nusselt number as function of $y^{*}$ position for different Reynolds numbers in the region of $-0.5 \geq y^{*} \geq 4.5$ will be analyzed.

In this region, the behaviour of $\mathrm{Nu}$ in the top channel is the same for both geometries.

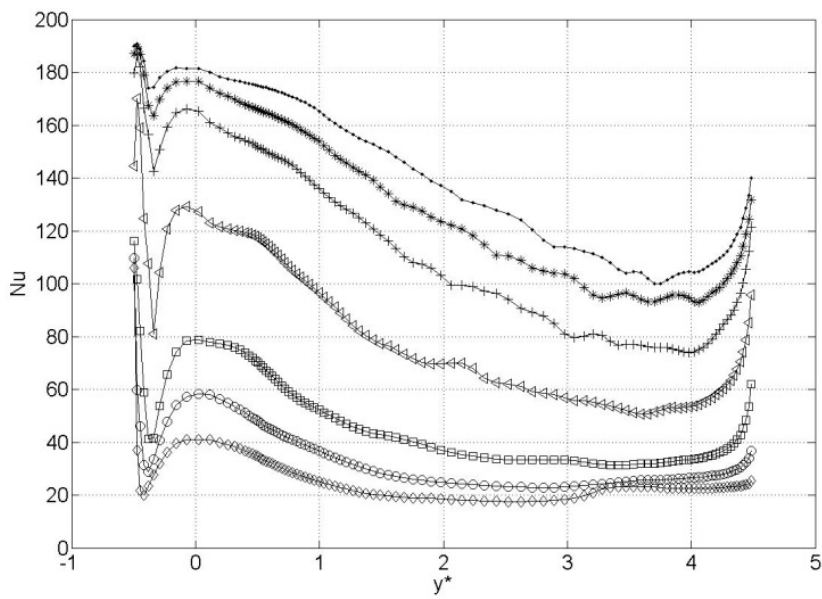

Figure 5: Mean Nusselt number vs. y* in the top channel for the open geometry with $\Delta \mathrm{T}=10^{\circ} \mathrm{C}$ for $\operatorname{Re}=300(\diamond), \operatorname{Re}=600\left({ }^{\circ}\right)$, $\operatorname{Re}=1160(\square), \operatorname{Re}=3000(\triangleright), \operatorname{Re}=5790(+), \operatorname{Re}=8000(*)$, $\operatorname{Re}=10000(\cdot)$.

The mean Nusselt number is calculated by averaging along the $x^{*}$ direction. In the top channel, both in closed and open geometry there is a strong $\mathrm{Nu}$ fluctuation in the region that matches the input channel at $\mathrm{y}^{*}=[-0.5,0.5]$. The aforementioned fluctuation has at least a sharp minimum followed by a maximum and coincides with the jet impinging onto the plate produced when the fluid enters to the cavity. From the maximum the $N u$ values decreases until $y^{*}=4$ to increases near the passage channel $\left(y^{*}=4.5\right)$, this behaviour is verified for $R e$ greater than $R e=1160$. For $R e=300$ and 600 the behaviour described above is only maintained until $y^{*}=1$. 
Unlike what happens in the top channel, in the bottom channel can be seen remarkable differences in the behaviour of the Nusselt number between the geometrical configurations studied as figs. 6 show.

In the closed geometry can be seen that at $y^{*}=4.5$ presents the maximum values of the Nusselt number to decrease when reaching the area of the channel output $y^{*}=0.5$.
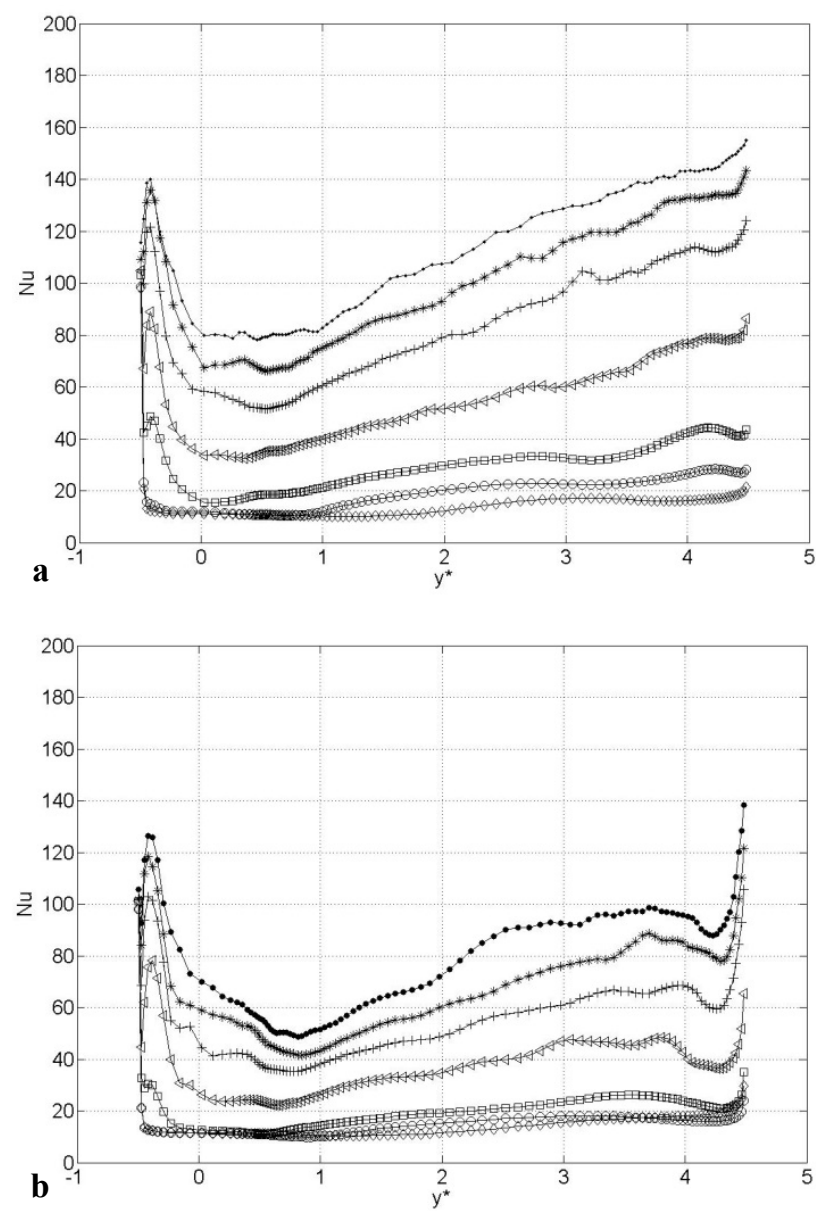

Figure 6: $\quad \mathrm{Nu}$ vs. $\mathrm{y}^{*}$ in the bottom, $\Delta \mathrm{T}=10^{\circ} \mathrm{C}$, for $\mathrm{Re}=300(\diamond), \mathrm{Re}=600$ $(\circ), \operatorname{Re}=1160(\square), \operatorname{Re}=3000(\triangleright), \operatorname{Re}=5790(+), \operatorname{Re}=8000(*)$, $\operatorname{Re}=10000(\cdot)$. a Open geometry; b Closed geometry. 
The decrease rate depends on $R e$, being greater for higher Reynolds numbers. This behaviour is consistent with the temperature fields shown in fig. 6 . For low $\operatorname{Re}\left(300\right.$ and 600) shows that from $y^{*}=1$ different mean $N u$ converges to the same value $(N u=12)$ and in $y^{*}=-0.5$ presents a great growth due to the wall boundary condition, as in the top channel. For $R e \geq 1160$ in the region $-0.5 \geq$ $y^{*} \geq 0$ presents a fluctuation similar to that observed in the top channel, although has a sharper minimum and a narrow maximum. In the open geometry (fig. 6a) the region $4 \geq y^{*} \geq 4.5$ shows a minimum for the $R e$ greater than 1160. This minimum coincides with the recirculation zone seen in fig. 2 . Furthermore, the $N u$ values at $y^{*}=4.5$ are lower than those observed in the closed geometry. In region $1 \geq y^{*} \geq 4$ the mean $N u$ decreases and $1 \geq y^{*} \geq 0$ there is a new minimum sharper for the higher $R e$. This minimum matches with the hot zone seen in fig. 3a. The behaviour at $-0.5 \geq y^{*} \geq 1$ is similar to the one obtained for the closed geometry.

\section{Conclusions}

The temperature fields in both geometries show the influence on the choice of $R e$. In the top channel, the mean temperature field is the same for both geometries at least until the passage channel for all Re studied. In the bottom channel, for the closed geometry, the presented flow structures are responsible for cooling the temperature in some regions of the lower side of the plate. The lower temperature coincides with the vortices location while the higher temperatures are located in the channel output zone. While in the open geometry the small, weaker, vertical structures presented result in a higher value of temperature field along the channel.

The heat transfer between the PCB and the fluid has been studied by means of the computation of the $N u$ number. The behaviour of the mean Nusselt numbers as function of $y^{*}$ position for different $R e$ is the same for both geometries in the top channel. In the bottom channel this behaviour is different depending on the geometry and the variations are consistent with the flow structures present in each case. The greater heat transfer is obtained in the top channel for both geometries. Instead in the bottom channel the heat transfer is greater (up to $80 \%$ more) in the closed geometry than in the open one.

The results obtained indicate that the cooling of the PCB is more efficient for the closed geometry. Small changes in the PCB geometry modify the velocity flow field allowing the formation of new vortical structures in the bottom channel of the PCB that enhance the heat transfer between the fluid and the plate.

\section{Acknowledgements}

This study was financially supported by the Spanish Ministry of Science and Innovation and FEDER under projects DPI2009-11204. 


\section{References}

[1] Ferziger J. Pèric M, Computational methods for fluid dynamics, SpringerVerlag, 2002.

[2] J.M. House, C. Beckermann, T. F. Smith: Numerical Heat Transfer, Part A, Vol. 18 (1990), p. 213.

[3] M. Y. Ha, M. J. Jung, Y. S. Kim: Numerical Heat Transfer, Part A, Vol. 35 (1999), pp.1574.

[4] J. R. Lee, M. Y. Ha: International Journal of Heat and Mass Transfer, Vol. 49 (2006), p. 2684.

[5] J. Bigorra, F.J. Sanchez, I. Cuesta, J. Herrero, J. Giralt \& F.X. Grau: Advanced Computational Methods in Heat Transfer VI, (2000).

[6] G. Usera, A. Vernet, J.A. Ferré, Use of time resolved PIV for validating LES/DNS of the turbulent flow within a PCB enclosure model, Flow Turbulent Combust. 77, pp. 77-95, 2006.

[7] F.P. Incropera, Convection heat transfer in electronic equipment cooling. Journal of Heat Transfer, 110, pp. 1097-1111, 1988.

[8] G. Usera, A. Vernet, J.A. Ferré, A Parallel Block-Structured Finite Volume Method for Flows in Complex Geometry with Sliding Interfaces, Flow Turbulent Combust., 81, pp. 471-495, 2008.

[9] T. Lehnhauser, M. Schafer, Improve linear interpolation practice for finitevolume schemes on complex grids. Int. J. Numer. Methods Fluids, 38, pp. 625-645, 2002.

[10] Cabeza, C., Sarasúa G., Martí, A., Bove, I., Varela, S., Usera, G., Vernet, A.: Influence of coaxial cylinders on the vortex breakdown in a closed flow, European Journal of Mechanics - B/Fluids, 29 (3), pp. 201-207, 2010.

[11] Cabeza. C., Varela. J., Bove. I., Freire. D., Martí. A., Sarasúa. L.G., Usera. G., Montagne. R., Araujo. M., Two-layer stratified flows over pronounced obstacles at low-to-intermediate Froude numbers. Phys. Fluids, 21 (4), pp. 102-112, 2009.

[12] U. Bückle, M. Pèric, Numerical simulation of buoyant and thermocapillary convection in a square cavity, Numerical Heat Transfer, Part A, 21, pp. 121-141, 1992.

[13] G. Usera, Master Thesis, L.E.S. Simulación numérica de grandes vórtices Aplicación al flujo en torno a un cilindro cuadrado. Montevideo, 2001.

[14] Varela. S., Usera. G., Vernet. A., Ferré. J.A., Numerical simulation of flow structures and temperature distribution in a Printed Circuit Board enclosure model at moderate Reynolds numbers, International Journal of Thermal Sciences. 70, pp. 1-9, 2011. 